\title{
EL PAPEL DE LA NUEVA PDVSA EN LA LUCHA CONTRA LA POBREZA Y LA EXCLUSIÓN SOCIAL EN VENEZUELA ${ }^{1}$
}

\author{
Por: Neritza Alvarado²; Maryoly Molero ${ }^{3}$; Ingrid Olmos ${ }^{4}$; José Soto ${ }^{5}$
}

\section{RESUMEN}

Se estudia el papel de Petróleos de Venezuela Sociedad Anónima (PDVSA, la principal empresa estratégica del Estado en este país) en la lucha contra la pobreza-exclusión social, en tanto organismo promotor, financista y ejecutor de programas y proyectos sociales, junto con el poder comunal, desde el año 2003 cuando pasa a control directo del gobierno bolivariano. El objetivo del trabajo es discernir algunos logros y limitaciones de esa intervención, a través de dos proyectos de desarrollo social ejecutados por PDVSA-Occidente (Maracaibo y Costa Oriental del Lago), en dos comunidades de los municipios Maracaibo y Lagunillas del estado Zulia, entidad-región petrolera por excelencia. Se toma como base la percepción de los actores involucrados, tanto de parte de la empresa como de las comunidades. Se realiza una breve revisión documental,

1. Este trabajo deriva del proyecto de investigación intitulado "El rol social de las empresas estratégicas del Estado venezolano en la lucha contra la pobreza-exclusión: los casos de PDVSA y CORPOZULIA", diseñado y coordinado por la profesora Neritza Alvarado, al cual estuvieron adscritas dos tesis de la Escuela de Sociología de la Universidad del Zulia, dirigidas por la misma profesora, a cargo de los bachilleres, hoy egresados, coautores del artículo.

2. Doctora en Estudios del Desarrollo, MSc en Desarrollo Social. Socióloga. Profesora titular e investigadora de la Universidad del Zulia, Venezuela, adscrita al Instituto de Investigaciones de la Facultad de Ciencias Económicas y Sociales, y a la Escuela de Sociología. Email: neritzaalvarado@ yahoo.es

3. Socióloga, estudiante de la Maestría en Desarrollo Social. Universidad del Zulia. Email: maryolymolero@yahoo.com

4. Socióloga, estudiante de la Maestría en Planificación Social. Universidad Nacional Experimental Simón Rodríguez. San Carlos-Cojedes. Email: ingridelena86@hotmail.com

5. Sociólogo, estudiante de la Maestría en Recursos Humanos. Universidad Nacional Experimental Rafael María Baralt. Estado Zulia. Email: josenriquesoto84@hotmail.com.

Artículo recibido: 28 de enero de 2015.

Aprobación definitiva: 29 de mayo de 2015 
especialmente de la normativa que le prescribe ese nuevo rol social a PDVSA; y un trabajo de campo dentro de una investigación cualitativa etnográfica, con uso del método estudio de casos, observación directa y entrevistas semiestructuradas en profundidad a informantes clave: gerentes de desarrollo social y coordinadores de los proyectos; voceros de los consejos comunales y beneficiarios directos. Según los informantes, el papel de PDVSA ha sido decisivo en el mejoramiento de las condiciones de vida aunque no suficiente para erradicar la pobreza; los beneficiarios se sienten incluidos y tienen expectativas positivas hacia el futuro, no obstante admiten limitaciones como retraso en los proyectos, débil comunicación industria-comunidad, escasa participación de ésta y ausencia de contraloría social.

Palabras clave:Venezuela, empresas estratégicas del Estado, PDVSA, rol social, pobreza-exclusión.

JEL: I32, P42.

\title{
THE NEW PDVSA ROLE IN FIGTH AGAINST POVERTY AND SOCIAL EXCLUSION IN VENEZUELA
}

\author{
By: Neritza Alvarado, Maryoly Molero, Ingrid Olmos, José Soto
}

\begin{abstract}
This paper analyzes the active role played by Petróleos de Venezuela SA (PDVSA, the main strategic State enterprise), since 2003, when it became directly controlled by the bolivarian government, as an entity promoter, financier and implementer of diverse social programs and projects, together with the communal power, aimed to combat poverty and exclusion in the country. So, PDVSA plays unpublished social functions and therefore a new historical role, as part of its responsibility for the development of the endogenous-socialist model. The objective of this work is to discern some achievements and limitations of this intervention (until 2011), through two social development projects executed by PDVSA-West (Maracaibo Lake and East Coast) in two communities of extreme poverty of Maracaibo and Lagunillas Municipalities, traditional oil region, in Zulia State, Venezuela. It builds on the perception of the involved actors, both, the company and communities. A literature review is made, especially the legislation that prescribes that new role to PDVSA; and a field work in a qualitative ethnographic research, using the case study method, direct observation and semi-structured in-depth interviews to key informants: social development managers and project coordinators; spokesmen communal councils and direct beneficiaries. According to the informants, the social role of PDVSA has been an instrumental in improving the living conditions, although it's not enough
\end{abstract}


to eradicate poverty; the beneficiaries feel included and have positive expectations for the future, however they allow limitations as delayed projects, weak industry-community communication, low participation of the community and lack of social control.

Keywords: Venezuela, strategic state enterprises, PDVSA, social roles, poverty and exclusion.

JEL: I32, P42.

\title{
NOVO PAPEL NA LUTA CONTRA A PDVSA - A POBREZA EA EXCLUSÃO SOCIAL NA VENEZUELA
}

\author{
Por: Neritza Alvarado, Maryoly Molero, Ingrid Olmos, José Soto
}

\section{RESUMO}

Este artigo examina o papel na luta contra a pobreza e a exclusão social da Petroleos de Venezuela SA (PDVSA, a principal empresa estatal estratégica neste país), como entidade promotora, financiadora e executora de programas e projetos sociais, juntamente com o poder comunal, desde 2003, quando ela passa o controle direto do governo bolivariano. O objetivo deste trabalho é discernir algumas conquistas e limitações dessa intervenção, através de dois projetos de desenvolvimento social implementados pela PDVSA-Oeste (de Maracaibo y da Costa Leste do Lago), em duas comunidades dos municípios Maracaibo e Lagunillas do estado Zulia, importante região petrolífera. O estudo se baseia na percepção dos atores envolvidos, da empresa y das comunidades.Uma breve revisão da literatura e documentos é feita, especialmente dos regulamentos que prescrevem o novo papel social para PDVSA.Também foi realizado um trabalho de campo numa pesquisa qualitativa etnográfica, utilizando o método Estudo de Casos, a observação direta e entrevistas semi-estruturadas, em profundidade, a informantes-chave: gerentes de desenvolvimento social e coordenadores dos projectos; porta-vozes dos conselhos comunitários e beneficiários diretos. De acordo com os informantes, o papel da PDVSA tem sido fundamental para melhorar as condições de vida mas não é suficiente para erradicar a pobreza; os beneficiários se sintam incluídos e têm expectativas positivas para o futuro, no entanto, eles admitem limitações como projetos adiados, fraco comunicação indústria-comunidade, baixa participação desta e falta de controle social.

Palavras-chave: Venezuela, empresas estratégicas do Estado, PDVSA, papel social, a pobreza-exclusão.

JEL: I32, P42. 


\section{INTRODUCCIÓN}

La empresa estatal Petróleos de Venezuela Sociedad Anónima (PDVSA), rectora de la industria de ese tipo en el país, fue creada en el año 1975, su antecedente es la Corporación Venezolana de Petróleo (CVP), fundada en 1960. Por tanto, el contexto histórico, económico y político que le da origen es la llamada democracia representativa y el modelo de crecimiento hacia dentro o de sustitución de importaciones, durante el cual las funciones y actividades de la industria petrolera fueron de índole estrictamente económica (exploración, explotación, exportación, refinación y transporte de petróleo y otros hidrocarburos derivados), fuente principal del Producto Interno Bruto y del ingreso fiscal del país, de carácter totalmente rentista y distribución regresiva e inequitativa.

Pese a que PDVSA es el resultado de la nacionalización de la industria, tras la cual pasa a propiedad nominal del Estado venezolano, continuó en gran medida bajo la égida del capital transnacional, articulado a las élites internas de poder económico-financiero y político; nunca se apartó del objetivo de fomentar el crecimiento económico del país, no formó parte de su quehacer enfrentar las desigualdades sociales ni intervenir de manera directa en el desarrollo social, lo cual fue acentuado con el advenimiento del neoliberalismo a fines de los 80s y durante la década de los 90s (Luzardo, 1994).

A partir de 1999, con el inicio del gobierno llamado Bolivariano o Quinta República, presidido por el Comandante, disidente de las Fuerzas Armadas, Hugo Chávez Frías, electo por una amplia mayoría en sufragios democráticos celebrados en diciembre de 1998, se pone en marcha un nuevo modelo de desarrollo, planteado en el discurso como humanista, integral e incluyente, contrario y alternativo al neoliberalismo, preocupado por articular todas las esferas de desarrollo, así como la política económica con la política social, decidido a darle respuestas a los problemas que aquejaban a la población venezolana, principalmente a hacerle frente a la pobreza, a la exclusión y a lograr la participación ciudadana en los asuntos públicos, fomentando la organización social, sobre todo de carácter popularcomunitaria (Alvarado, 2010).

No obstante, en el primer cuatrienio de gestión (1999-2002), la lógica revolucionaria del nuevo modelo no logró penetrar en la lógica capitalista, conservadoraliberal de PDVSA, quien a decir del Presidente Chávez funcionaba como un Estado dentro de un Estado, por su holgada autonomía; con una lógica de empresa privada más que de empresa pública, cuyo esquema de administración de la alta gerencia de la empresa, en su opinión, estaba enquistada en un modelo tecnocrático, contrario a las ideas emancipadoras del esquema de izquierda bolivariana, al punto de escenificar paros llamados "cívicos" y posteriormente, entre diciembre de 2002 y febrero de 2003, un paro económico (petrolero y comercial) patronal, junto a otros sectores de oposición política al gobierno, que veían amenazados sus intereses corporativos privados y exigían la renuncia del presidente (Chávez, 2001).

Como parte del propósito declarado de colocar el ingreso fiscal y los activos nacionales al servicio de todos los ciudadanos, especialmente de los más vulnerables, el gobierno bolivariano inició a partir del año 2003 un proceso de reestructuración de las empresas estratégicas del Estado venezolano, bajo la rectoría del 
Ministerio de Energía y Petróleo (MENPET), asignándoles un papel central en la planificación, promoción, conducción y financiamiento del desarrollo social, mediante el combate de la pobreza y la exclusión social, conforme a los principios del modelo de desarrollo endógeno-socialista, de un estado de derecho, de justicia y de equidad social, pluriétnico y pluricultural, y del proyecto de país de democracia participativa-protagónica, prescrito en la Constitución Bolivariana y en los planes de desarrollo económico-social de la nación.

En este contexto surge la "Nueva PDVSA" en el año 2003, una vez que el gobierno logró vencer el paro petrolero patronal, denominación que alude al hecho que, en el nuevo esquema de intervención estatal, esta empresa trasciende su rol económico-financiero tradicional y ejecuta un rol social inédito, a través del apoyo a los programas de lucha contra la pobreza-exclusión y a la participación comunitaria, a fin de fomentar el empoderamiento ciudadano, el poder popular y, en general, el desarrollo social (MINCI, 2004).

El objeto de este trabajo es analizar la gestión social de la Nueva PDVSA, en una investigación microsociológica (realizada durante los años 2010 y 2011, que recupera información desde el año 2003, de la cual este artículo constituye un apretado resumen), tomando como caso de estudio su intervención en dos comunidades del estado Zulia en situación de pobreza crítica y exclusión social, a saber: "Pinto Salinas" y "Tierra Santa” (ubicadas en los municipios Maracaibo y Lagunillas, respectivamente), mediante dos proyectos de desarrollo social integral ejecutados por PDVSA-Occidente (Municipio Maracaibo y Sub-región Costa Oriental del Lago de Maracaibo), con los objetivos de: 1) identificar su rol social, mediante el desempeño de la Gerencia de Desarrollo Social en el período 2003-2011; 2) discernir los principales logros y limitaciones de la gestión social de PDVSA según la opinión de los actores involucrados (ejecutores directos, líderes comunales y beneficiarios de los proyectos).

Se trata de una investigación cualitativa-etnográfica, que aplica el método Estudio de Casos. Como tal no aplica la técnica de la encuesta ni instrumentos cuantitativos precodificados sino la observación participante y la entrevista semiestructurada, grabada, abierta y en profundidad. A través de éstas se indagó información sobre las siguientes categorías de análisis: a) el conocimiento de los informantes sobre la "Nueva PDVSA", su nuevo rol social y los proyectos de desarrollo social que ejecuta a fin de combatir la pobreza-exclusión; b) su conocimiento y opinión sobre la ejecución de los proyectos sociales de PDVSA y sobre su coordinación o no con otras instituciones públicas y con las organizaciones comunitarias; c) $\mathrm{Su}$ apreciación sobre el desempeño social de la empresa (logros e impacto social sobre la pobreza-exclusión; y sus limitaciones); d) Sus expectativas y perspectivas sobre la intervención social de la "Nueva PDVSA".

Tomando en cuenta que los estudios sobre el tema son escasos, especialmente con un enfoque cualitativo de investigación, este trabajo recupera la perspectiva de los actores involucrados sobre una realidad que se está viviendo en Venezuela y procura dar cuenta, de la manera más objetiva posible, de cómo la industria petrolera, a través de su máxima representante, concibe hoy su papel en la sociedad venezolana y ha venido modificando su intervención dentro del modelo de 
desarrollo endógeno y de socialismo del siglo XXI; indagando hasta qué punto está contribuyendo de una manera efectiva a combatir la pobreza, la exclusión y las desigualdades sociales en el país.

\section{EL ROL NORMATIVO DE LA NUEVA PDVSA EN EL DESARROLLO SOCIAL}

Como se apuntó arriba, la respuesta inmediata del presidente Hugo Chávez en el segundo momento de su gestión (a partir el año 2003) para encarar la coyuntura crítica, de alta polarización y conflictividad política, que se venía acentuando, fue plantear un nuevo modelo productivo a través del rescate de fabricas cerradas, el impulso al Desarrollo Endógeno, el lanzamiento de las misiones sociales, el fomento al desarrollo comunal como herramientas para la estabilidad política y social a través de nuevas formas de organización y producción social (Álvarez, 2009).

En ese proceso post-paro petrolero el gobierno adquiere control sobre la empresa estatal PDVSA. Bajo la defensa de la soberanía petrolera, enfocada en impulsar la productividad, se edifica la "Nueva PDVSA", pasando a ser, según el discurso oficial, una empresa básica subordinada al Estado venezolano, profundamente comprometida con el desarrollo integral del país y con el auténtico dueño del petróleo: "el pueblo venezolano".

El Presidente Chávez propuso que se le cambiara parcialmente la denominación y se le llamara "Petróleos de Venezuela Socialista", otorgándole al entonces titular del Ministerio de Energía y Petróleo, a la vez presidente de PDVSA, Rafael Ramírez, la tarea de realizar los estudios legales para abandonar la denominación de Sociedad Anónima heredada del modelo capitalista, con el argumento de que la empresa estatal debía estar acorde con el nuevo proyecto de país. No obstante, aún mantiene su antigua personalidad jurídica.

Así, la Nueva PDVSA debe coadyuvar con el desarrollo del país, contribuyendo con mano de obra, recursos financieros y otros recursos en el desarrollo de los programas sociales, en atención al artículo 5 de la Ley de Hidrocarburos, según el cual es obligación de PDVSA que los ingresos derivados de la actividad petrolera sean empleados no sólo en fondos de estabilización macroeconómica sino en salud, educación y participación social. Tal como lo expresa su sitio web, es deber de PDVSA: "alinear las estrategias y los planes de hidrocarburos con el plan de desarrollo social de la nación, desarrollar el negocio de los hidrocarburos, fortalecer la OPEP, impulsar el desarrollo endógeno y el capital nacional, mejorar las bases y composición de las reservas, valorizar la cesta de exportación, reorientar la internacionalización e industrialización de los hidrocarburos, fortalecer las actividades medulares" (PDVSA, 2005: 3).

La estrategia "plena soberanía petrolera" apunta a promover actividades petroleras y no petroleras, con el lema "siembra petrolera", plan cuya premisa es que la renta será para beneficio del pueblo soberano. De esta manera, el rol de la nueva PDVSA pasa por acatar también el artículo 15 de la Ley de Hidrocarburos, según el cual debe cumplir con los planes de desarrollo de la nación, siendo así "una empresa que no se detiene y mantiene su compromiso de ejecutar políticas colectivas y no individuales. La responsabilidad de PDVSA es alcanzar una visión de desarrollo social sustentable en todo su entorno" (Rodríguez, 2004:7; MINCI, 2004:8). 
Por ello, según se afirma en el sitio web de PDVSA, ésta promueve los núcleos de desarrollo endógeno a través de su filial Palmaven, de manera que los esfuerzos sociales e institucionales se concentren en un espacio social específico, en comunidades concretas con condiciones sociales, culturales y productivas particulares, enfatizando la atención en poblaciones que legítimamente demandan su participación en la construcción de una región, con parte de los beneficios generados por el petróleo. De esta manera, a su propio entender, el Ministerio de Energía y Petróleo y PDVSA contribuyen con la creación de un modelo nacional de desarrollo, donde las comunidades de menores recursos puedan creer en el bienestar, tener calidad de vida y ser parte activa de una misma nación (PDVSA, 2005).

Bajo este modelo de desarrollo económico y social, en el año 2006 nacen los consejos comunales, definidos como órganos integradores de carácter comunitario, generadores de capital humano y social, trabajando de manera articulada con las instituciones del Estado en cuanto a las políticas públicas y sociales, y en particular con PDVSA en los proyectos financionados y/o ejecutados por ésta (Min. Comunas, 2009).

\section{ACCIONES DEL ESTADO VENEZOLANO EN PRO DEL NUEVO ROL DE PDVSA}

En materia legal, el gobierno se avocó a partir de 2003 a hacer cumplir los artículos 5 y 15 de la Ley de Hidrocarburos, antes referidos. En segundo lugar, planteó y concretó la reforma a la Ley del Banco Central de Venezuela (BCV), que entró en vigencia en julio de 2005, y autorizó el empleo parcial de las reservas internacionales para financiar proyectos estratégicos y los nuevos programas sociales, a partir de la creación del Fondo de Desarrollo Nacional (FONDEN) y del Fondo para el Desarrollo Económico y Social (FONDESPA, de PDVSA). Se creó una Ley de Contribuciones Especiales por precios extraordinarios del petróleo -por ganancia súbita- (PDVSA, 2005).

Este fue el recurso estratégico que el gobierno encontró para lograr que los ingresos excedentarios (no previstos en el presupuesto nacional), fuesen depositados, una parte en el FONDEN -para planes estratégicos de desarrollo- y otra en el FONDESPA, de donde provienen básicamente los recursos que financian a las misiones, a los consejos comunales y a otros proyectos sociales. Es decir, que éstos no derivan del presupuesto ordinario de los ministerios sino directamente de PDVSA (Alvarado, 2012). De ahí que el presidente de esta empresa expresara que "Petróleos de Venezuela es una empresa nacional que está al servicio del Estado venezolano, un servidor público, su brazo técnico y operativo, que logre explotar, producir y desarrollar ese recurso para colocarlo en beneficio del pueblo, del interés nacional” (Ramírez, 2005: 4).

En ese sentido, el MENPET conjuntamente con PDVSA presentó en 2005 la política petrolera denominada "Plena Soberanía Petrolera", definida como una política nacional, popular y revolucionaria; argumentando que es nacional, ya que busca el control general de la actividad petrolera, amparándose en la Constitución de la República Bolivariana de Venezuela; popular, porque la participación del pueblo es considerada fundamental para el desarrollo de esta política, y revolucionaria, 
porque afirma que procura la forma de redistribuir la renta petrolera con beneficios al país y a sus ciudadanos, con equidad y justicia (PDVSA, 2005).

Como otra de las acciones también se formuló el "Plan Siembra Petrolera (20052012)", cuya misión, según el ministro respectivo, no es sólo ejecutar tareas petroleras sino también no petroleras, siendo una orden del gobierno que se invierta el $10 \%$ de ese plan en proyectos estructurales, a ser ejecutados por PDVSA con apoyo de la población venezolana (Ramírez, 2006: 8).

También se cuenta entre las decisiones oficiales la creación de las Empresas de Producción Social (EPS), planteadas como "entidades económicas dedicadas a la producción de bienes o servicios en las cuales el trabajo tiene significado propio, no alienado y auténtico, no existe discriminación social en el trabajo y de ningún tipo de trabajo" (Ramírez, 2006: 8). Con esta iniciativa se procura que los trabajadores de la empresa petrolera nacional y de empresas privadas se unan para crear sus propias empresas de producción, para prestarle servicios necesarios directos a PDVSA e incluso a las empresas privadas, para convertirse en exportadores de bienes y servicios, para ayudar en el desarrollo nacional y además para hacer aportes en obras sociales, con arreglo al Plan Siembra Petrolera (Ramírez, 2005).

Otra estrategia es la creación de los "Distritos Sociales", petroleros y gasíferos, es decir, grupos constituidos territorialmente, con carácter productivo, ecológico y social, con el objetivo expreso de que los procesos de exploración, producción y refinación se traduzcan en apoyos a la educación, salud, y lucha contra la pobreza. Estos distritos sociales funcionan en los estados con actividad petrolera, no obstante, según el ministro, no se desatienden aquellos territorios sin esa actividad, pues el desarrollo sería impulsado en todo el país, a través de la filial Palmaven (Ramírez, 2005).

Otro aspecto destacado en los discursos del Presidente de PDVSA, que ésta trata de fomentar, es el desarrollo de un nuevo perfil del gerente público, definido como un actor capaz, que esté en concordancia con los planes estratégicos establecidos, que además del negocio petrolero, conozca las comunidades, se involucre e interactúe con ellas, y que sea un gerente integral: "nuestro compromiso es gerenciar una empresa nacional en tiempos de revolución” (Ramírez, 2005: 2).

Así, PDVSA se convierte en un motor fundamental del desarrollo social, fomentando el nuevo modelo socio productivo con proyectos de distinta índole: agrícola, industrial, prestación de servicios, construcción de infraestructuras, financiamiento a programas de salud, educación, vivienda, alimentación, para lograr una vinculación de los recursos de los hidrocarburos, con la economía nacional, de acuerdo a lo establecido en el Plan de Desarrollo Económico y Social de la Nación 2007-2013 y en el Proyecto Nacional Simón Bolívar, para el mismo período. Incluso para atender las necesidades alimentarias de la población, fue creada la empresa Productora Venezolana de Alimentos (PDVAL), como una nueva filial de PDVSA, que coordinaría la Misión MERCAL (Mercado de Alimentos), que se ejecuta desde el año 2003. Ello da cuenta de la diversificación del campo de acción de la Nueva PDVSA. 
Como parte de las iniciativas en este sentido, PDVSA agregó una nueva gerencia a su estructura organizativa-operativa: la Gerencia de Desarrollo Social, a finales del 2003 y principios de 2004, activándola totalmente en el año 2005, siendo su función principal, según sus propios voceros, "alinear los planes y los proyectos con la política social del Estado, facilitar y fortalecer el rol de PDVSA como agente de cambios en los procesos de desarrollo económico y social del país” (PDVSA, 2009:10).

De acuerdo con los documentos oficiales, la Gerencia de Desarrollo Social de PDVSA está orientada a la consolidación de una nueva estructura social, justa e incluyente, en la cual prevalezcan los valores de solidaridad e igualdad social que se traducen en nuevas formas de producción, apropiación y distribución de los recursos económicos. Según sus representantes, el principal punto focal de PDVSA es "gestionar sus recursos, salvaguardar la capacidad de la compañía para continuar como una empresa en marcha, con el propósito de seguir siendo la fuerza y motor para el desarrollo nacional y la palanca para la transformación del país". (PDVSA, 2009:10).

Un indicador ilustrativo de su nuevo rol es la magnitud del gasto social de la empresa: en el año 2003 PDVSA contribuyó, según el ministro Ramírez, con 6.300 millones de dólares para la construcción de escuelas, universidades, viviendas, carreteras, aportes a las misiones sociales. En 2005, PDVSA destinó 4 mil millones de dólares al área social, en apoyo a las misiones de inclusión, y se destinaron al Fondo de Infraestructura 12 mil millones, derivados de regalías, 8 mil millones del impuesto sobre la renta y 2 mil millones fueron colocados en el Fondespa (Ramírez, 2006: 8). Durante el año 2009 PDVSA ejecutó un plan de inversión, con amparo en sus dividendos, de 600 mil millones de dólares de aportes al FONDEN y 2,483 millones de dólares a programas de desarrollo social, producto de la gestión de los últimos años y de los resultados operacionales y financieros de la empresa (PDVSA, 2009:10).

El mismo ministro Ramírez, en 2011, afirmó que según la Memoria y Cuenta de 2010 del Despacho de Energía y Petróleo, en materia de gasto social PDVSA hizo una inversión de 83.834 millones de dólares, de los cuales 27.930 se destinaron a Fondos de Inversión; 29.459 al Fonden y 25.995 a las necesidades fundamentales del pueblo, entre ellas 2.610 millones a alimentación. En ese año, la Gerencia de Desarrollo Social de PDVSA tenía como foco principal la construcción de 150 mil viviendas, conjuntamente con el Ministerio de Hábitat y Vivienda; como parte de la llamada Gran Misión Vivienda Venezuela, siendo la meta 250 mil casas para el año 2012 y 2 millones en 6 años (MENPET, 201: 7). A través de la Gerencia Social, PDVSA viene ejecutando estos proyectos en todo el país.

\section{EL ROL DE PDVSA-OCCIDENTE EN LA LUCHA CONTRA LA POBREZA-EXCLUSIÓN, EN MARACAIBO Y LA COSTA ORIENTAL DEL LAGO, ESTADO ZULIA}

En las entidades federales, PDVSA motoriza el Plan de Aceleración del Desarrollo Regional (PADRE), creado por el Ejecutivo Nacional para dinamizar los proyectos gubernamentales en los estados y regiones del país. En el caso del Zulia, estado región petrolero por excelencia, aparte del apoyo a las misiones, a los con- 
sejos comunales y otras actividades sociales, PDVSA-Occidente ha intervenido de otras formas, por ejemplo, con labores de electricidad en el Municipio Miranda; obras educativas en los Municipios Mara, San Francisco, Insular Almirante Padilla, Machiques de Perijá, Colón, Jesús María Semprún y la Cañada de Urdaneta. En el área ambientalista se ha ocupado del saneamiento de las costas del lago de Maracaibo, entre otras actividades (MENPET, 2011).

En el municipio Maracaibo, PDVSA-Occidente, a través de su Gerencia de Desarrollo Social, ha intervenido en el área de la salud con la remodelación de la Maternidad “Dr. Castillo Plaza”, así como en mejoras y cambio de administración del Hospital Coromoto, que antes era privado y atendía la póliza de salud del personal de la vieja PDVSA, entre otras instituciones, ahora atiende también (y de manera gratuita) a los pacientes remitidos por la Misión Barrio Adentro, a la población de escasos recursos de Maracaibo, del Zulia y de otros estados vecinos(MENPET, 2011).

En el Municipio Maracaibo, una de las iniciativas que destaca es el proyecto “Ciudad Educativa Integral Simón Rodríguez”, en la comunidad Pinto Salinas, sector "El Marite”, de la Parroquia Venancio Pulgar. Por otra parte, en la Costa Oriental del Lago de Maracaibo, PDVSA-Occidente ha desarrollado, entre otros, el proyecto de reforestación de Bachaquero, capital del Muncipio Valmore Rodríguez; y el Proyecto “Huertos Familiares", en la comunidad Tierra Santa, de la parroquia Libertad del Municipio Lagunillas, o Distrito Social Lagunillas, en el argot de PDVSA (MENPET, 2011).

La Gerencia de Desarrollo Social de PDVSA-Occidente trabaja con las comunidades a través de una estructura organizativa a la cual denomina "Maracaibo Divisional”, que no sólo atiende a las comunidades del municipio Maracaibo, sino que tiene personal asignado a lo que denominan "Municipios Indígenas" (Villa del Rosario, Machiques, Urdaneta, Almirante Padilla, Guajira (Páez) y Mara); “Tomoporo” (municipio Baralt y estado Trujillo); “Lagunillas” (Municipios Lagunillas y Valmore Rodríguez); “Cabimas”(Cabimas, Santa Rita y municipio Miranda); “Tía Juana”(Lago, Municipio Simón Bolívar); y "San Francisco” (Municipios San Francisco, Urdaneta y Jesús Enrique Lossada) (PDVSA-Occidente, Gerencia de Desarrollo Social, 2011).

En cada uno de estos municipios hay personal encargado, que son los delegados para darle vida a los proyectos, a través de lo que PDVSA denomina "cadena de valores", es decir, las tareas que deben ejecutar para alcanzar el objetivo de mejorar la calidad de vida de la población menos solvente. Estas cadenas de valores estarían orientadas en función de: 1) el fortalecimiento del poder popular (ayudar a organizar consejos comunales a las comunidades que no lo hayan conformado), en concordancia con el Ministerio del Poder Popular para las Comunas; es decir, enseñar a las comunidades a organizar su consejo comunal; 2) la gestión comunitaria (interrelacionar a las comunidades con el Ministerio y otros entes gubernamentales); 3) inversión social: usar los procedimientos de la empresa para la compra de bienes y servicios, de las compras efectuadas por la empresa debe emerger un porcentaje que será destinado a los proyectos sociales; y 4) Proyectos socioproductivos: la formulación y ejecución de proyectos que generen empleo y mejoren la calidad de vida de las comunidades (Morales, 2011). 
Siendo numerosos los proyectos de desarrollo social ejecutados por PDVSAOccidente, en esta investigación se toman como casos de estudio el Proyecto "Ciudad Educativa Integral Simón Rodríguez", en la comunidad Pinto Salinas (Municipio Maracaibo); y el Proyecto "Huertos Familiares", en la comunidad Tierra Santa (Municipio Lagunillas), el primero correspondiente al sector o área urbana; $\mathrm{y}$ el segundo al sector rural.

\section{A) PERFIL DE LAS COMUNIDADES Y DE LOS PROYECTOS DE PDVSA-OCCIDENTE}

Pinto Salinas, es una comunidad fundada en 197, en la Parroquia Venancio Pulgar del Municipio Maracaibo, ubicada al oeste de la ciudad de Maracaibo, capital del estado Zulia. Al momento de la realización del trabajo de campo de este estudio, en el año 2011, estaba en ejecución un proyecto de sustitución de ranchos (viviendas improvisadas) por casas, iniciado en el año 2006, aún no concluido, por lo cual todavía se observaban ranchos. Se trata de una comunidad en pobreza crítica, con serias carencias de servicios públicos (no cuenta con red de cloacas, ni aseo urbano, ni gas, ni telefonía residencial).

Dispone del servicio de electricidad, logrado por la Asociación de vecinos que existía antes de la creación de los actuales consejos comunales. Asimismo, la comunidad no cuenta con carreteras asfaltadas, ni con aceras. Sus calles de arena, sin pavimentar, dificultan el acceso en épocas de lluvia. La acumulación de agua trae consigo enfermedades, queja recurrente de sus pobladores. En ese momento, esta comunidad no poseía instituciones educativas, con lo cual los niños y adolescentes debían trasladarse a pie a los colegios de localidades vecinas.

Sin embargo, la comunidad tiene en su haber un establecimiento donde funciona un complejo cultural, llamado "Casa Soñadores de Venezuela", donde los niños reciben clases de música, artes escénicas, tecnología e innovación, crecimiento familiar, estudios bolivarianos, escritores venezolanos, etc.,; y disponen de un comedor y una psicóloga. Esta Casa cultural fue ejecutada y es administrada por la empresa Desarrollos Urbanos S.A (DUCOLSA), adscrita a PDVSA, y la Asociación de Ayuda a la Infancia Necesitada (ADAIN).

Dichas actividades están dirigidas a niños en edad preescolar, pues hasta entonces sólo funcionaba un "Simoncito", que atendía alrededor de 500 infantes. Se estaba a la espera de la entrega correspondiente de la primera fase del proyecto “Ciudad Educativa Integral Bolivariana Simón Rodríguez”, también bajo la responsabilidad de ejecución DUCOLSA. La comunidad cuenta con un Consejo Comunal, conformado en el año 2006 y está dotada de un consultorio popular de la Misión Barrio Adentro. Asimismo, la misión Mercal (alimentos básicos subsidiados por el gobierno) funciona a pequeña escala en algunos hogares del sector. Pinto Salinas "es una comunidad pequeña, relativamente segura, carente de muchos servicios, pero sus vecinos tienen expectativas positivas respecto de los proyectos de PDVSA.

Por su parte, Tierra Santa, es una comunidad rural, en pobreza crítica, ubicada en la Parroquia Libertad del Municipio Lagunillas del estado Zulia. Cuenta con una Casa de Alimentación financiada por PDVSA, un ambiente de la Misión Robinson (programa de alfabetización de adultos y culminación de la educación primaria), 
una Iglesia Evangélica, tres bodegas para autoconsumo y una casa de acopio para distribución y venta de bombonas comunales (gas doméstico).

Posee escasas viviendas fabricadas con bloques, predominan los ranchos construidos por los mismos habitantes. A su alrededor tiene eminentes riesgos que ponen en peligro la vida de sus pobladores, como una planta de gas cerca del sector, tendidos eléctricos de alta tensión, tomas ilegales de electricidad realizadas por medio de alambres y palos de madera improvisados, vialidad en pésimo estado (lo cual dificulta el acceso al sector), delincuencia y la presencia alarmante de tráfico y consumo de drogas, así como prostitución adulta e infantil, dada a la situación de pobreza crítica de sus habitantes.

Se pudo observar que en la comunidad las habilidades y destrezas son muy dispersas, sin embargo, la mayor fortaleza es la agricultura de pequeña escala, además de albañilería, costura y repostería ya que en este sector son muchas las personas que trabajan por cuenta propia, un número significativo de personas se dedican a la siembran y otros a la pesca. "Tierra Santa” posee múltiples problemas, desde su constitución como comunidad, donde la falta de planificación y crecimiento descontrolado ha caracterizado su composición; asimismo adolece de atención de los organismos competentes en las áreas de salud, educación, vivienda, infraestructura comunal, entre otras. No obstante, tiene potencial en el área de siembra y agricultura.

\section{LOS PROYECTOS DE PDVSA EN EL ZULIA:“CIUDAD EDUCATIVA INTEGRAL SIMÓN RODRÍGUEZ" $y$ “HUERTOS FAMILIARES"}

El proyecto “Ciudad Educativa Integral Simón Rodríguez”, planificado para la comunidad Pinto Salinas, del Municipio Maracaibo, tiene como prerrequisito la ejecución del proyecto "Dignificación Revolucionaria El Marite", consistente en la sustitución de "ranchos" o viviendas improvisadas e inseguras por viviendas dignas. En un primer radio de acción se planteó la edificación de 1.074 casas.

El Proyecto Ciudad Educativa Integral tuvo como asignación un área de 58.000 metros cuadrados, a ser desarrollado en 4 fases: a) la construcción de un "Simoncito" (preescolar) y una escuela de educación especial; b) una escuela bolivariana; c) un liceo bolivariano y una escuela técnica robinsoniana; y d) una biblioteca, un auditorio, un comedor, un gimnasio y una plaza central.

El proyecto es financiado por PDVSA y ejecutado por DUCOLSA, con participación de la Fundación de Edificaciones y Dotaciones Educativas (FEDE) y la empresa PEMEGAS C.A., bajo el entendido tácito de participación de la comunidad a través de sus respectivos comunales. El proyecto fue aprobado por el presidente de la República en 2006, y él mismo colocó la primera fundacional en ese año, no obstante el inicio oficial de las obras fue en julio 2007; luego estuvo año y medio paralizado, reiniciándose en 2008.

PDVSA también apoya a esta comunidad en la recolección de basura, sirve de intermediaria entre el consejo comunal y la empresa constructora y contrata 
personas de la comunidad para el desarrollo de las obras, bajo la figura de empleo temporal y los criterios del desarrollo endógeno.

Por otra parte, el Proyecto Huertos Familiares forma parte del Megaproyecto denominado "Dignificando los Sectores con Pobreza Extrema", previsto para abarcar siete parroquias de la Costa Oriental del Lago de Maracaibo, entre ellas la parroquia Libertad, del Municipio Lagunillas. "Tierra Santa" es la primera comunidad de esta parroquia en ser seleccionada para el desarrollo de los "Huertos Familiares Socioproductivos", impulsado en 2007, como proyecto piloto. Se trata de un proyecto agroproductivo, ecológico y orgánico, financiado por PDVSA, con la finalidad de desarrollar las potencialidades productivas de la comunidad, para que el productor beneficiario pueda autoabastecerse y vender sus excedentes a precios solidarios dentro y fuera de su comunidad.

La adecuación inicial del suelo fue efectuada por la comunidad, para lo cual PDVSA, a través de la Fundación CIARA (Capacitación e Innovación para Apoyar la Revolución Agraria), dictó talleres a fin de crear cultura en cuanto a la siembra, adiestrando a las familias en la realización de los huertos. PDVSA donó las semillas iniciales y los materiales para la constitución de los huertos familiares y creó un centro de acopio para la distribución de los productos. Algunas semillas también fueron donadas por la Corporación de Desarrollo de la Región Zuliana (Corpozulia). Hoy se cosechan diversos rubros, tales como plátano, auyama, tomate, cebolla, frutas varias.

\section{RESULTADOS DE LA INVESTIGACIÓN: EL ROL SOCIAL DE PDVSA SEGÚN LOS ACTORES INVOLUCRADOS}

A continuación se presenta un resumen de los principales hallazgos del estudio realizado, ordenados con arreglo a las categorías de información exploradas, recordando que por tratarse de metodología cualitativa, los resultados no son presentados en forma de números, porcentajes o distribuciones de frecuencia estadística. Es necesario aclarar también que en el informe final de la investigación extensa de la cual hace parte este artículo, dichos resultados fueron documentados con abundantes testimonios textuales de los informantes, emanados de las entrevistas (no encuestas) realizadas, de los cuales muchos tuvieron que ser omitidos en la versión resumida publicable, dado que abultaban demasiado el texto, por lo cual los hallazgos son aludidos de una manera global. El documento monográfico in extenso (Alvarado, 2011) puede ser consultado en el Instituto de Investigaciones de la Facultad de Ciencias Económicas y Sociales de la Universidad del Zulia o comunicándose con los autores. Otros resultados complementarios pueden consultarse en las tesis de grado adscritas a este proyecto, disponibles en la escuela de Sociología de la Universidad del Zulia (Molero, 2011) y Olmos y Soto (2011).

\section{a) Conocimiento de los informantes sobre la "Nueva PDVSA", su nuevo rol social y los proyectos de desarrollo social para combatir la pobreza-exclusión.}

Los gerentes de desarrollo social y los voceros de los consejos comunales conocen suficientemente lo que es la "Nueva PDVSA" y su nuevo rol social de intervención en la lucha contra la pobreza-exclusión. Sin embargo, los beneficiarios de los 
proyectos tienen escaso conocimiento al respecto; muy genéricamente la asocian a las siguientes ideas: "aquella que ayuda al pueblo"; "ahora PDVSA es de todos"; "ayuda a la gente pobre", "ahora sí hay acceso a ella"; vinculando su nuevo rol social directamente a la figura del Presidente Chávez ("Chávez mandó ese proyecto pa’acá”; "Chávez es el único que se ha acordado de los pobres”).

\section{b) Conocimiento y opinión sobre la ejecución de los proyectos sociales de PDVSA, su coordinación con otras instituciones públicas y con las organizaciones comunitarias.}

Es débil el conocimiento de los beneficiarios sobre la participación de PDVSA en el desarrollo del país (en general); lo remiten a los proyectos que se están ejecutando en su comunidad, es decir, no lo asocian al nivel macro sino a su realidad micro, inmediata: "PDVSA ayuda a las comunidades": "da empleo, ayuda a las madres necesitadas", "ayuda a los enfermos con medicamentos". Los gerentes hablan de trabajo coordinado con la comunidad y otras instituciones; los beneficiarios ratifican que PDVSA trabaja con los Consejos Comunales y con las misiones sociales, pero es PDVSA quien más influye en la comunidad en cuanto a la toma de decisiones colectivas, es decir, el consejo comunal presenta rezagos en sus funciones.

\section{c) Apreciación sobre el desempeño de PDVSA (logros e impacto social sobre la pobreza-exclusión); y las limitaciones de su nuevo rol social}

Los beneficiarios tienen una valoración positiva de la intervención de PDVSA: "los proyectos sociales son buenos"; "los de PDVSA están haciendo un buen trabajo"; "antes PDVSA sólo era pa 'los ricos”, "nos ha cambiado los ranchos por casas", "nos recoge la basura", "nos da trabajo dentro de la comunidad con las obras", "los beneficios son directos". Asimismo, valoran positivamente el Proyecto Ciudad Educativa Integral: "los muchachos ya no tendrán que salir de la comunidad a estudiar afuera". Se sienten incluidos, es decir, tomados en cuenta por PDVSA, sin embargo, la participación e inclusión es asociada a la idea de recibir pasivamente el beneficio, de no quedarse fuera de los proyectos, no en términos de cooperación activa, en tanto sujetos de derecho.

No hay un voluntariado notorio y permanente que coadyuve con la empresa y con los consejos comunales en la consecución de los logros colectivos. No se ejecuta la contraloría social prevista en el proyecto, ni por parte de la comunidad ni por los funcionarios de PDVSA. La Gerencia de Desarrollo Social no realiza seguimientoevaluación del proyecto ni del alcance de sus objetivos. Según opinión de los beneficiarios, PDVSA no resuelve la pobreza pero ayuda, alivia las cargas de los más carenciados, las comunidades viven relativamente mejor ahora (no hay ranchos). Afirman que antes de PDVSA nadie más hizo nada por ellos en muchos años.

Entre las limitaciones asociadas al rol social de PDVSA se cuentan: retraso en las obras inherentes a los proyectos sociales, inasistencia reiterada de los ingenieros y técnicos de PDVSA al trabajo, falta de supervisión, "pago de vacuna" a delincuentes (una especie de "peaje" o "impuesto" como mecanismo de extorsión) para que los trabajadores de la empresa puedan entrar a las comunidades y cumplir con su trabajo; solapamiento de los Consejos Comunales (sobre-intervención de PDVSA en la comunidades); no hay una comunicación fluida y diálogo de saberes entre 
PDVSA y las comunidades; falta de organización en la ejecución de los trabajos; son frecuentes las quejas y reclamos de la comunidad por el desfase de los proyectos con relación al tiempo estimado de ejecución.

\section{d) Expectativas y perspectivas sobre la intervención social de la "Nueva PDVSA"}

Al momento de la ejecución del trabajo de campo en el año 2011, en ambas comunidades se esperaba que PDVSA culminara los proyectos en cuanto a servicios públicos: "las carreteras, las cloacas, el gas, que terminen las casas"; y "que cumplan con todas las obras educativas sin más retrasos". Había confianza en que PDVSA cumplirá sus compromisos. Pese a las limitaciones, se observó respaldo (aunque pasivo) a la intervención social de PDVSA en las comunidades. No obstante, según los testimonios de los informantes, las comunidades estudiadas no tenían la expectativa de que el gobierno o la industria petrolera venezolana, a través de su máximo representante, fuese capaz de acabar con la pobreza y la exclusión de la comunidad en otros aspectos, problemas que consideran muy complejos y precisan de la ejecución de "muchas otras cosas y proyectos", pero sí aspiraban en lo inmediato y en los años más próximos mejoras parciales y progresivas en su calidad de vida.

\section{CONCLUSIONES}

El papel de PDVSA en la lucha contra la pobreza-exclusión y en general en el desarrollo social del país, es reconocido como importante y positivo por los actores involucrados en los proyectos estudiados como referencia; los beneficiarios de éstos admiten cambios relativos favorables, no radicales, en sus condiciones de vida, considerando poco probable la erradicación de la pobreza y la exclusión social a partir de la intervención de PDVSA por medio de proyectos puntuales y aislados.

Si bien los esfuerzos de la industria no son considerados como suficientes para abatir dichos problemas, los beneficiarios se sienten socialmente incluidos al ser los destinatarios directos de los proyectos. En general repiten las consignas del gobierno: "ahora PDVSA es del Pueblo" o "PDVSA es de todos", sin mucho conocimiento del alcance de la intervención social de esta empresa petrolera. Pese a limitaciones (retraso en la ejecución de proyectos u obras inconclusas, débil comunicación entre la industria y la comunidad, escasa participación de ésta en los proyectos, falta de supervisión y de contraloría social, entre otras), los actores mantienen una expectativa positiva ante la gestión de PDVSA, especialmente los beneficiarios de los proyectos, quienes confían en el cumplimiento a futuro de los compromisos.

Según la investigación realizada, la intervención de PDVSA a través de los proyectos referidos contribuye escasamente a la organización, articulación y participación comunitaria. Destaca la apatía de los beneficiarios a insertarse de una manera dinámica y corresponsable en la acción colectiva. Ello indica que la gestión de políticas públicas y proyectos comunitarios liderados por PDVSA, a la luz de los casos aquí estudiados, no se traduce aún en el logro de un empoderamiento popular protagónico, como se espera dentro del proyecto de país vigente en Venezuela. 


\section{REFERENCIAS}

1. ALVARADO, CHACÍN Neritza (2012). El modelo endógeno socialista de Venezuela y sus estrategias de inclusión social. En: Revista de Ciencias Sociales. Vol. XVIII. N 4, pp. 641-656. Universidad del Zulia. Facultad de Ciencias Económicas y Sociales. Venezuela

2. ALVARADO, CHACÍN Neritza. (2011). Informe final del proyecto El rol social de las empresas estratégicas del Estado venezolano en la lucha contra la pobreza-exclusión: Ios casos de PDVSA y CORPOZULIA. (mimeo). Instituto de Investigaciones Económicas y Sociales. Universidad del Zulia.

3. ALVARADO, CHACÍN Neritza. (2010). La política social de lucha contra la pobreza y la exclusión en el gobierno de Hugo Chávez (1999- 2009). En: “Diez Años del Gobierno de Hugo Chávez". Editorial de la Universidad del Rosario. Bogotá. Colombia. pp. 345-364.

4. ÁLVAREZ, Víctor (2009). Venezuela: ¿Hacia dónde va el nuevo modelo productivo? Centro Internacional Miranda (Disponible en http://www.aporrea.org). Consultado: 10-01-2011.

5. CHÁVEZ, Hugo (2001). ¿Qué hacer con PDVSA? Discurso del Presidente de la República Bolivariana de Venezuela. Disponible en: http://www.minci.gob.ve/2001/02/discursosnuevapdvsa.pdf (Consultado: 07-09-2007).

6. LUZARDO, Gastón, (1994). La Apertura Petrolera, ¿alternativa ante la crisis?. EDILUZ. Universidad del Zulia, Maracaibo, Venezuela.

7. MINISTERIO DEL PODER POPULAR PARA LA ENERGÍA Y EL PETRÓLEO, MENPET (2011). Memoria y Cuenta. Año 2010. Disponible en: http://www.pdvsa.com (Consultado 09-03-2011).

8. MINISTERIO DEL PODER POPULAR PARA LA COMUNICACIÓN E INFORMACIÓN, MINCI (2004). La Nueva PDVSA:produciendo Bienestar para el Pueblo. Publicación del Ministerio. Caracas. MINCl. Consultado: 10-01-2011. Disponible en: http://www.minci.gob.ve/wp-content/uploads/downloads/2013/01/ folletolanuevapdvsa.pdf

9. MINISTERIO DEL PODER POPULAR PARA LAS COMUNASY MOVIMIENTOS SOCIALES (MIN.COMUNAS, 2010). Ley Orgánica de los Consejos Comunales 2009. Disponible en: www.mpcomunas.gob.ve

10. MORALES, RIXIO (2011). Gerente de Desarrollo Social de PDVSA-Occidente-Maracaibo. Entrevista realizada en esta investigación.

11. MOLERO, Maryoly (2011). El Rol Social de PDVSA-Occidente en la Comunidad "Pinto Salinas". Municipio Maracaibo, Estado Zulia. Tesis de Grado. Escuela de Sociología. Universidad del Zulia, Maracaibo (Tutoreado por: Profesora Neritza Alvarado).

12. OLMOS, Ingrid y SOTO, José (2011). El Rol Social de PDVSA-Occidente en la Comunidad "Tierra Santa". Municipio Lagunillas, Estado Zulia. Tesis de Grado. Escuela de Sociología. Universidad del Zulia, Maracaibo (Tutoreado por: Profesora Neritza Alvarado). 
13. PETRÓLEOS DE VENEZUELA, PDVSA (2005). La Nueva PDVSA. Disponible en: http://www.pdvsa.com (Consultado 20-06-2010).

14. PDVSA (2009). Informe de Gestión Anual 2009. Disponible en: http://www.pdvsa.com (Consultado 20-06-2010).

15. RAMÍREZ, Rafael (2005). Ministro del MENPET y Presidente de PDVSA. Nuestro Compromiso es gerenciar una empresa nacional en tiempos de revolución. MENPET. Discursos. Discurso $\mathrm{N}^{\circ} 5$ ( 11 de julio de 2005). Caracas.

16. RAMÍREZ, Rafael (2006). El Petróleo es la Plataforma de la Soberanía de Venezuela. MENPET. Discursos. Discurso $\mathrm{N}^{\circ} 4$ ( 19 de enero de 2006). Caracas.

18. RODRÍGUEZ ARAQUE, Félix (2004). Vicepresidente Ejecutivo de PDVSA. Discurso de fecha 24/08/2004. Disponible en: www.aporrea.org/actualidad/n19770.html (Consultado 20-06-2010). 